\title{
Silicon photonic crystal filter with ultrawide passband characteristics
}

\author{
Anna Baldycheva, ${ }^{1}$ Vladimir A. Tolmachev, ${ }^{2}$ Tatiana S. Perova, ${ }^{1, *}$ Yulia A. Zharova, ${ }^{2}$ \\ Ekaterina V. Astrova, ${ }^{2}$ and Kevin Berwick ${ }^{3}$ \\ ${ }^{1}$ Department of Electronic and Electrical Engineering, Trinity College Dublin, Dublin 2, Ireland \\ ${ }^{2}$ Ioffe Physical Technical Institute, Polytechnicheskaya 26, St. Petersburg, Russia \\ ${ }^{3}$ Department of Electronic and Communications Engineering, Dublin Institute of Technology, Kevin Street, Dublin 8, Ireland \\ ${ }^{*}$ Corresponding author: perovat@tcd.ie
}

Received January 26, 2011; revised April 6, 2011; accepted April 9, 2011;

posted April 11, 2011 (Doc. ID 141239); published May 9, 2011

\begin{abstract}
We report on what is believed to be the first example of an ultrawide, bandpass filter, based on a high-contrast multicomponent one-dimensional Si photonic crystal (PC). The effect of the disappearance of a limited number of flat stopbands and their replacement with extended passbands is demonstrated over a wide IR range. The passbands obtained exhibit a high transmission of $92 \%$ to $96 \%$ and a substantial bandwidth of $1800 \mathrm{~nm}$, which is spectrally flat within the passband. The multicomponent PC model suggested can be applied to the design of any micro- or nanostructured semiconductor or dielectric material for application across a wide spectral range. (C) 2011 Optical Society of America

OCIS codes: $220.0220,230.0230,230.3990,250.5300,260.0260$.
\end{abstract}

Optical filters, which pass desired wavelengths and reject unwanted wavelengths, are key components in Si-based optical integrated circuits [1]. In recent years, photonic crystal (PC) structures have attracted much attention due to their potential application to optical filtering over a wide IR range [2-4]. As a result of the photonic bandgap (PBG) effect, PCs are traditionally used as broadband Bragg mirrors. The creation of narrow passband peaks within the PBG by introducing disorder within the crystal components, or by forming a Fabry-Perot resonator, allows a variety of Si PC bandpass filters to be realized [5]. However, the ultranarrow bandwidth of the bandpass filters reported are often unsuitable for application to integrated photonic devices, such as antireflective structures or tunable polarizers, where ultrawide bandpass characteristics are essential. Recently, we reported a new type of one-dimensional (1D) PC structure, with wide transmission bands (TBs), using a multicomponent PC model [6]. Thermal oxidation, which can be exploited to tune the PBGs in grooved Si structures [7], is technologically attractive as a method for the fabrication of multicomponent PCs, since it has good growth isotropy, particularly for narrow, deep trenches or pores, and precise thickness control. In this Letter, we report, for the first time to our knowledge, on theoretical and experimental results from $\mathrm{Si}$ bandpass filters derived from grooved $\mathrm{Si}, 1 \mathrm{D}$ PCs, with a thermally grown, regular $\mathrm{SiO}_{2}$ component. For some wavelengths, the limited number of spectrally flat stopbands (SBs) vanish and are replaced by ultrawide TBs. The TBs obtained demonstrate a substantial bandwidth (>200 nm) and a negligible number of ripples within the bands.

Figure 1 is a schematic of the device. The $1 \mathrm{D}$ band-stop filter is originally composed from two materials, $\mathrm{Si}$ and air, with thicknesses $d_{\mathrm{Si}}$ and $d_{\text {air }}$, following deposition of $\mathrm{SiO}_{2}$ of thickness $d_{\mathrm{ox}}$ on the Si sidewalls. The filling fraction value is defined as $f_{\mathrm{Si}}=d_{\mathrm{Si}} / a$, where $a$ is the lattice constant. The refractive indices are taken as $n_{\mathrm{Si}}=3.42, n_{\mathrm{ox}}=1.5$, and $n_{\text {air }}=1$ for the wavelength range $3-10 \mu \mathrm{m}$, corresponding to frequencies of
100-30 THz. In our simulations, the imaginary part of the refractive indices, the absorption, is assumed to be $k=0$.

Thermal oxidation of $\mathrm{Si}$ results in the consumption of a thickness of $\mathrm{Si}$ equal to $44 \%$ of that of the grown oxide, while the remaining $56 \%$ of the oxide thickness grows above the original silicon surface.

Thus, the thickness of the Si walls and the air gap width are reduced as the thickness of the oxide layer, $d_{\mathrm{ox}}$, increases. Therefore, the filling fraction of the resulting three-component device will be reduced by the value $\Delta f_{\mathrm{Si}}=\left(2 \cdot 0.44 \cdot d_{\mathrm{ox}}\right) / a$. Only three $\mathrm{Si}$-air paired layers are necessary in order to demonstrate SB formation and possible replacement with passbands, making the device extremely compact and, therefore, probably exhibiting low transmission losses. An in-plane design, in which the SBs' structure is etched into the Si substrate, allowing the incorporation of optical fiber in-plane, eases the integration process and facilitates the construction of a high-quality filter. Using a transfer matrix method, one can accurately simulate a $1 \mathrm{D} \mathrm{Si-air} \mathrm{stack} \mathrm{for} \mathrm{plane} \mathrm{wave}$ propagation. We use normalized frequency, $\mathrm{NF}=a / \lambda$, units and, therefore, the results obtained can be applied to a wide range of structure sizes, including micro- and nanostructures. Note that all the optical characteristics presented in this study have been simulated for normal

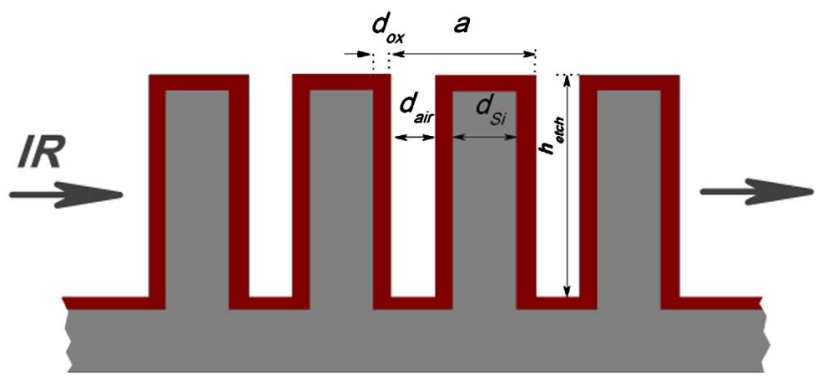

Fig. 1. (Color online) Schematic of a bandpass filter design viewed as a PBG mirror with thermally grown oxide on top. The number of periods $=3$. The etching depth is denoted as $h_{\text {etch. }}$. 

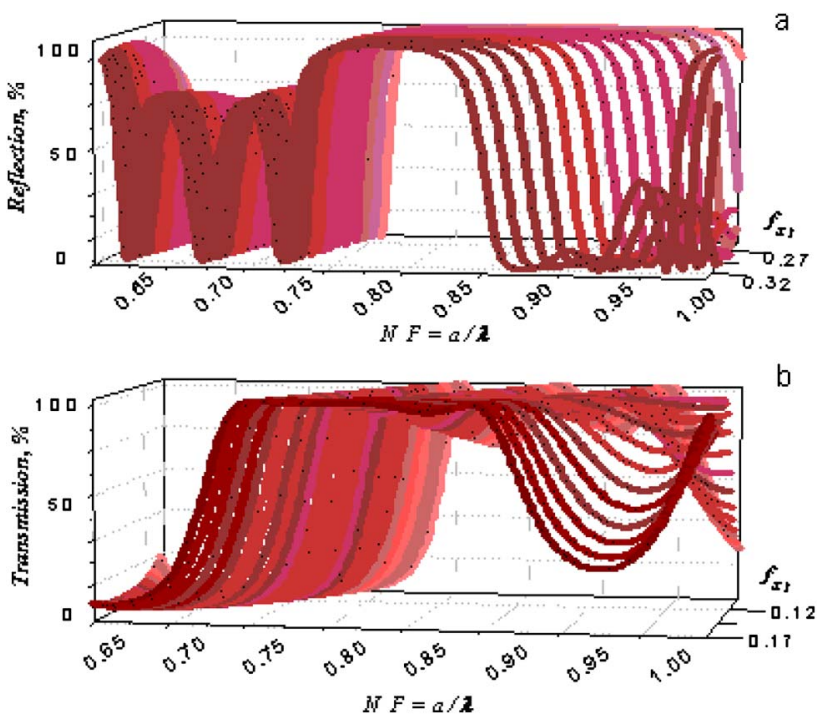

Fig. 2. (Color online) 3D representation of (a) flattop SBs centered at $0.8 \mathrm{NF}$ in the range of filling fractions $f_{\mathrm{Si}}=0.25-0.33$ before oxidation and (b) flattop passbands centered at the same frequency calculated for the same device after oxidation in the range $f_{\mathrm{Si}}=0.10-0.17$. The $\mathrm{SiO}_{2}$ thickness is $d_{\mathrm{ox}}=0.18 a$.

incidence of light and $n=1$ for both the incoming and outgoing media. The reflection, $R$, spectra for the original, two-component structure in the range of filling fractions from 0.25 to 0.33 are demonstrated in Fig. 2(a). The device calculated can operate as a reflective filter, with a wide SB centered at $\lambda_{c}=0.8 \mathrm{NF}$. Now, let us consider the impact on this particular SB caused by the insertion of an $\mathrm{SiO}_{2}$ component of thickness, for example, $d_{\mathrm{ox}}=0.18 a$. In Fig. 2(b), transmission spectra, $T$, are illustrated for the structure after thermal oxidation over the new range of filling fractions $f_{\mathrm{Si}}^{*}=f_{\mathrm{Si}}-\Delta f_{\mathrm{Si}}$, varying from 0.10 to 0.17 . It is apparent from the $T$ spectra that wide TBs (with $T>99 \%$ ) have totally replaced the SBs. The structure obtained can now operate as a bandpass filter centered on the same wavelength, $\lambda_{c}$.

As demonstrated earlier, one of the best approaches to the design of optical elements based on PC structures over a wide $f_{\mathrm{Si}}$ range is the gap map (GM) method [4]. GMs are based on the calculation of the $R$ and $T$ spectra over a wide range of $f_{\mathrm{Si}}$ values, say, from 0.01 to 0.5 ,

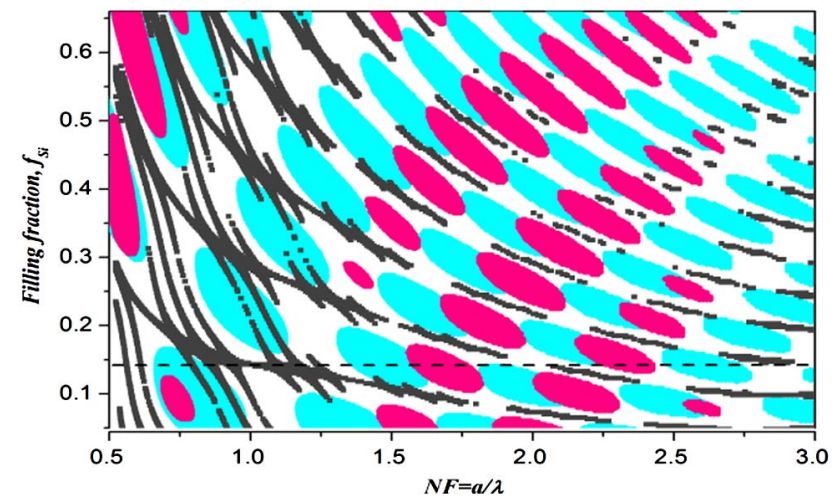

Fig. 3. (Color online) GMs for a two-component PC filter with optical contrast $n=3.42 / 1$ (light cyan) and for a three-component PC filter with thermally grown $\mathrm{SiO}_{2}$ of thickness $d_{\mathrm{ox}}=$ $0.18 a$ (light magenta). The map of TBs (black curves) is presented for the three-component PC.

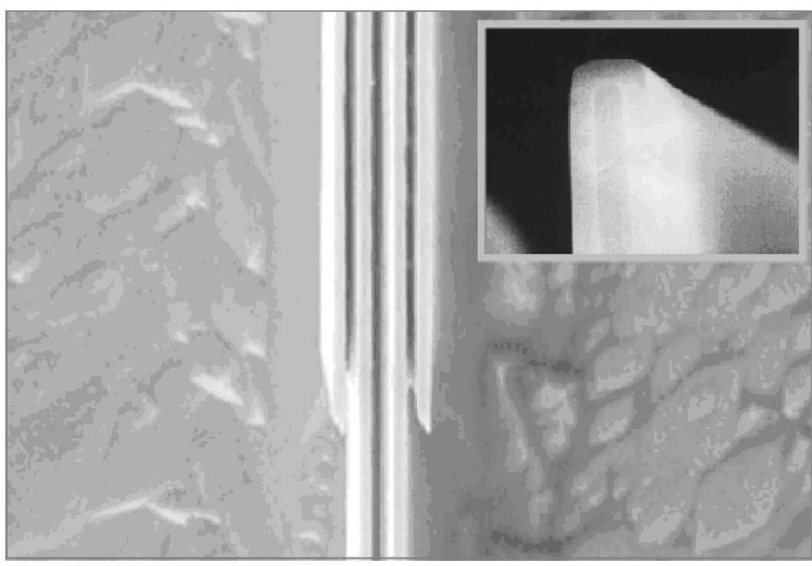

Fig. 4. Scanning electron microscope image of PC filter based on grooved $\mathrm{Si}$ with thermally grown $\mathrm{SiO}_{2}$ of thickness $d_{\text {ox }}=0.72 \mu \mathrm{m}$. The lattice period $a=4 \mu \mathrm{m}$. The number of periods $=3$.

followed by the selection of regions of wavelength, $\lambda$, where $R$ and $T$ are over 99\%. Figure 3 shows the maps for the structure discussed earlier, over a wide range of NF from 0.5 to 3.0. The GM of the SBs for the original two-component structure is also presented for comparison purposes. It is apparent that the introduction of regular $\mathrm{SiO}_{2}$ layers in the device, (Fig. 1), has affected the formation of the SBs significantly. The limited number of high-order SBs have vanished over the full range of $f_{\mathrm{Si}}$. In addition, regions of high transparency, with $T>99 \%$, have appeared over the same wavelength region. It is also clear that the widest TBs are observed for filling fraction values less than 0.5 , which actually correspond to more realistic structures for fabrication. Note that the SBs determined for the two-component PC structure are not suppressed over the entire NF range (Fig. 3 , light cyan regions).

Let us use the maps calculated for the fabrication of a real bandpass optical filter with an ultrawide passband centered on a wavelength of $4.2 \mu \mathrm{m}$. Because of the
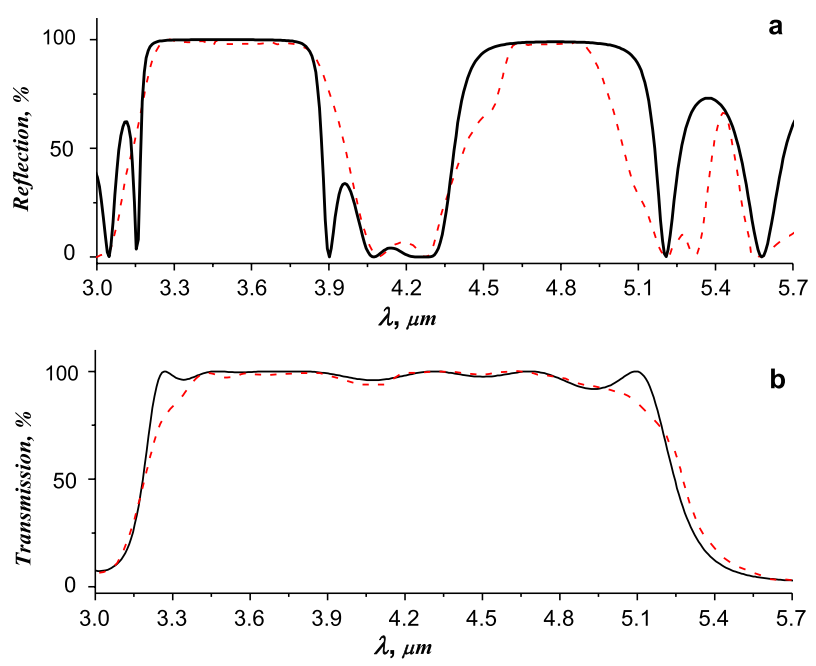

Fig. 5. (Color online) Experimental (red dashed line) and calculated (black solid line) (a) $R$ spectra and (b) $T$ spectra demonstrating the transformation from stopbands to passband at an operational wavelength of $\lambda_{c}=4.2 \mu \mathrm{m}$. 


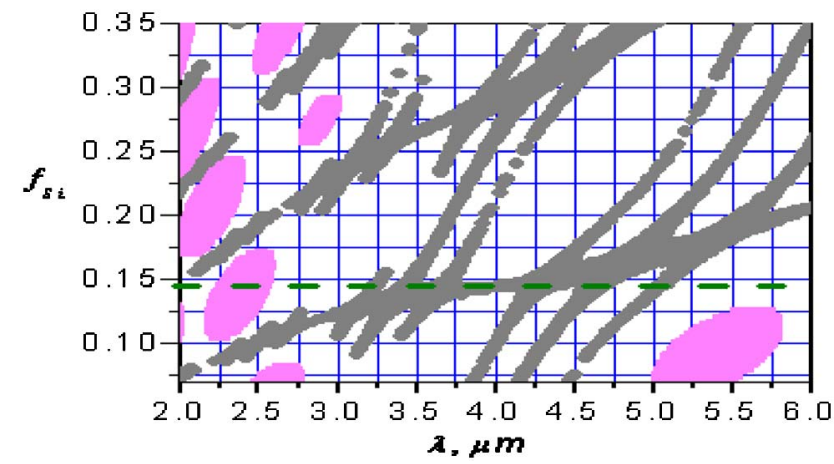

Fig. 6. (Color online) GM (light magenta) and map of TBs (dark gray) calculated for the fabricated filter over a limited range of filling fractions, $f_{\mathrm{Si}}$.

strong IR absorption band of $\mathrm{SiO}_{2}$ around $9 \mu \mathrm{m}$, dispersion of the refractive $\left(n_{\mathrm{ox}}(\lambda)\right)$ and the absorption $\left(k_{\text {ox }}(\lambda)\right)$ indices of the $\mathrm{SiO}_{2}$ have been taken into account [8]. It was found that if the lattice constant $a$ is $<5 \mu \mathrm{m}$, then the ranges of transparency for the filter obtained are outside this $\mathrm{SiO}_{2}$ absorption band. For ease of fabrication, let us choose a lattice period of $a=4 \mu \mathrm{m}$. According to the TB map from Fig. 3 (black curves), the final device should have a filling fraction $f_{\mathrm{Si}}^{*}=0.145$ and, therefore, a final Si-wall thickness of $d_{\mathrm{Si}}=f_{\mathrm{Si}}^{*} \cdot a=0.58 \mu \mathrm{m}$. In order to fabricate the initial $\mathrm{Si}$ grooved structure, we used wet anisotropic etching of (110) $\mathrm{Si}$ [9]. This process generates a mirrorlike quality for the $\overline{\mathrm{S} i}$ sidewalls [10], which creates a very pronounced flattop band in the transmission spectrum $T$. The values of the thicknesses $d_{\mathrm{Si}}$ and $d_{\mathrm{ox}}$ were also optimized. The etch depth was $15 \mu \mathrm{m}$, which was necessary for the investigation of the fabricated device over a wide IR range using Fourier transform IR microspectroscopy, where the minimum possible beam spot is $\geq 10 \times 10 \mu \mathrm{m}$. Following the etch, the grooved $\mathrm{Si}$ structure was thermally oxidized at $T=900{ }^{\circ} \mathrm{C}$ for $500 \mathrm{~min}$, in order to grow a smooth oxide layer of thickness, $d_{\mathrm{ox}}=0.18 a=$ $0.72 \mu \mathrm{m}$, on the Si sidewalls (Fig. 4). The inset shows a cross section of the vertical $\mathrm{SiO}_{2}-\mathrm{Si}-\mathrm{SiO}_{2}$ wall. Thickness control of the $\mathrm{SiO}_{2}$ layers was performed on a witness sample using spectroscopic ellipsometry. Reflection and transmission spectra measurements were performed using a Bio-Rad (now Varian, USA) FTS 6000 Fourier transform IR spectrometer equipped with a UMA-500 IR microscope. The aperture of the focused beam was $15 \times 15 \mu \mathrm{m}^{2}$, and the spectral resolution was $8 \mathrm{~cm}^{-1}$ (as described in [9]).

Figure 5 shows experimental $R$ and $T$ characteristics compared with the simulated spectra. There is good agreement between the experimental results and the simulations. Note that the original grooved structure already has two SBs centered on 3.5 and $4.8 \mu \mathrm{m}$ so the device operates as a band-stop filter [Fig. 5(a)]. Following thermal oxidation, these SBs are replaced by an ultrawide TB from 3.3 to $5.1 \mu \mathrm{m}$. The device obtained can operate as a bandpass filter [Fig. 5(b)]. Utilization of thermally grown $\mathrm{SiO}_{2}$ in the fabrication of the multicomponent PC device has resulted in the appearance of a number of ripples, with a depth of $4 \%$ to $8 \%$, on the otherwise flattop transmission. However, for all practical purposes these ripples can be ignored. So, in summary, the bandpass filter shown exhibits an ultrawide transmission region centered at $4.2 \mu \mathrm{m}$, with transmission values of between $92 \%$ and $96 \%$. Additional details can be seen in Fig. 6, where a map of the SBs and TBs for the fabricated sample are shown over the range of filling fractions, $f_{\mathrm{Si}}$, from 0.05 to 0.35 . The dashed line indicates the TBs and SBs for the structure. It is obvious that the filling fraction, $f_{\mathrm{Si}}$, deviation corresponding to the passbands of the same bandwidth and $\lambda_{c}$ is up to 0.015 . Therefore, the fabrication tolerance can be easily extracted from the TB map and is $60 \mathrm{~nm}$ for this example.

To conclude, the design of a new type of Si bandpass optical filter with a wide bandwidth has been demonstrated. The operation of the device proposed is based on the transparency effect obtained in a grooved Si structure following the introduction of an additional regular layer, such as $\mathrm{SiO}_{2}$. The GM approach has been demonstrated as a core design tool for these devices, allowing the estimation of the fabrication tolerances to a high degree of accuracy. A 1D PC bandpass filter with an ultrawide bandwidth has been fabricated. Experimental spectra from the device agree well with our theoretical model. Optical filters possessing wide stopbands together with wide passband characteristics, antireflective structures and polarizers integrated into photonic devices can be realized using the proposed technique. Our recent investigations have demonstrated that the wide TBs are maintained with oblique incidence of light from air and possess omnidirectional properties. Because of space limitations in this Letter, these results were not included here and are the subject of a future publication.

This work has been supported by the International Centre for Graduation Education in Micro \& Nano Engineering (ICGEE) Programme, funded by the Irish Research Council for Science, Engineering, and Technology (IRCSET), Ireland; the Russian Foundation for Basic Research (RFBR), project 09-02-00782, and a grant for Support of Leading Scientific Schools (NSh-33062010.2), funded by the Russian government.

\section{References}

1. M. Missiroli, Silicon-Based Microphotonics: from Basics to Applications (IOS, 1999).

2. E. Yablonovitch, Phys. Rev. Lett. 58, 2059 (1987).

3. S. John, Phys. Rev. Lett. 58, 2486 (1987).

4. J. D. Joannopoulos, S. G. Winn, and R. D. Meade, Photonic Crystals, 2nd ed. (Princeton University, 1995, 2008).

5. W. R. Jameroz, R. Kruzelecky, and E. I. Haddad, Applied Microphotonics (CRC Press, 2006).

6. A. Baldycheva, V. Tolmachev, T. Perova, and K. Berwick, IEEE Photon. Technol. Lett. 23, 200 (2011).

7. G. Barillaro, S. Merlo, and L. M. Strambini, IEEE J. Sel. Top. Quantum Electron. 14, 1074 (2008).

8. E. D. Palik, Handbook of Optical Constants of Solids (Academic, 1998).

9. V. A. Tolmachev, E. V. Astrova, J. A. Pilyugina, T. S. Perova, R. A. Moore, and J. K. Vij, Opt. Mater. 27, 831 (2005).

10. Y. Uenishi, M. Tsugai, and M. Mehregany, J. Micromech. Microeng. 5, 305 (1995). 